\title{
Constraints on non-Newtonian gravity and axionlike particles from measuring the Casimir force in nanometer separation range
}

\author{
G. L. Klimchitskaya, ${ }^{1,2}$ P. Kuusk, ${ }^{3}$ and V. M. Mostepanenko $\circledast^{1,2,4}$ \\ ${ }^{1}$ Central Astronomical Observatory at Pulkovo of the Russian Academy of Sciences, \\ Saint Petersburg 196140, Russia \\ ${ }^{2}$ Institute of Physics, Nanotechnology and Telecommunications, Peter the Great Saint Petersburg \\ Polytechnic University, Saint Petersburg 195251, Russia \\ ${ }^{3}$ Institute of Physics, University of Tartu, W. Ostwaldi 1, Tartu 50411, Estonia \\ ${ }^{4}$ Kazan Federal University, Kazan 420008, Russia
}

(Received 22 January 2020; accepted 1 March 2020; published 19 March 2020)

\begin{abstract}
We obtain constraints on the Yukawa-type corrections to Newton's gravitational law and on the coupling constant of axionlike particles to nucleons following from the experiment on measuring the Casimir force between a Au-coated microsphere and a silicon carbide plate. For this purpose, both the Yukawa-type force and the force due to two-axion exchange between nucleons are calculated in the experimental configuration. In the interaction range of Yukawa force exceeding $1 \mathrm{~nm}$ and for axion masses above $17.8 \mathrm{eV}$, the obtained constraints are much stronger than those found previously from measuring the lateral Casimir force between sinusoidally corrugated surfaces. These results are compared with the results of other laboratory experiments on constraining non-Newtonian gravity and axionlike particles in the relevant interaction ranges.
\end{abstract}

DOI: 10.1103/PhysRevD.101.056013

\section{INTRODUCTION}

Many extensions of the Standard Model predict the existence of light scalar and pseudoscalar particles which play an important role in the current concepts of the world, but still remain to be discovered experimentally. Among these particles, one could mention arion, graviphoton, dilaton, goldstino, axion, and other axionlike particles, moduli, chameleon, etc. [1-10]. An exchange of such particles between the constituents of two macrobodies results in novel macroscopic forces in addition to the familiar electromagnetic and gravitational interactions. Thus, an exchange of one light scalar particle leads to the Yukawa-type interaction potential [11], whereas the interaction potential due to an exchange of one pseudoscalar particle between two fermions depends on their spins $[12,13]$. It is interesting to note that a simultaneous exchange of two pseudoscalar particles coupled to fermions by means of the pseudoscalar Lagrangian results in the spin-independent interaction potential $[12,13]$. However, for fermions possessing the pseudovector coupling to

Published by the American Physical Society under the terms of the Creative Commons Attribution 4.0 International license. Further distribution of this work must maintain attribution to the author(s) and the published article's title, journal citation, and DOI. Funded by SCOAP . axions, the form of effective potential due to two-axion exchange remains unknown [14].

The possible existence of light scalar particles and associated with them Yukawa-type forces between macroscopic bodies is of great importance, because it is equivalent to the modification of Newton's law of gravitation at short separations where this fundamental law is not tested experimentally with sufficient precision [11]. We emphasize also that the Yukawa-type corrections to Newtonian gravity have been predicted $[15,16]$ in extradimensional physics assuming the low-energy compactification scale of the order of $1 \mathrm{TeV}[17,18]$. For these reasons, great pains were taken to detect the Yukawa-type force which may manifest itself in different experiments or at least to constrain its strength and interaction range as severely as possible. The latter goal was reached in the gravitational experiments of Eötvos and Cavendish type [19-22], measurements of the normal and lateral Casimir forces [23-33], and in experiments on neutron scattering [34-39].

The Yukawa-type correction to the Newtonian gravitational potential appears also in the post-Newtonian approximation of the scalar-tensor theory of gravity $[40,41]$. The constraints on this correction at the length scale of about astronomical unit were obtained using radio links with the Cassini spacecraft [41,42].

Various possibilities of observing the pseudoscalar axionlike particles are equally important for both particle physics and astrophysics and cosmology (see, e.g., reviews [43-45]). 
It has been known that axions help to resolve the problems of lacking strong $C P$ violation in $\mathrm{QCD}$ and the large electric dipole moment of a neutron. These particles are also considered the most probable candidates for the constituents of dark matter. For the mass range of our interest, the most important searches of axionlike particles are based on the Cavendish-type experiments [46,47], experiments measuring the Casimir force [48-55], and measurement of forces between protons in the beam of molecular hydrogen [56], which allow one to obtain competitive laboratory constraints on the coupling constants of axions to nucleons.

In this paper, we consider the hypothetical interactions arising from the exchange of scalar and pseudoscalar particles and obtain constraints on them which follow from recent measurements of the Casimir force between a goldcoated microsphere and a silicon carbide plate [57]. It has been known that in high-precision measurements of the Casimir interaction made in vacuum the minimum separation distance between the test bodies was more than $160 \mathrm{~nm}[26,27,58]$. Rather competitive constraints on the Yukawa force at a very short interaction range [25] have been obtained, however, from a less precise ambient measurement of the Casimir force between two crossed cylinders performed at separations from 20 to $100 \mathrm{~nm}$ [59]. Over a period of time, these constraints were the strongest ones in the interaction range $\sim 10 \mathrm{~nm}$. In more recent times, they were replaced by stronger constraints following from the experiment on neutron scattering [37] and from measurements of the lateral Casimir force between sinusoidally corrugated surfaces $[29,60,61]$. In the latter case, the closest approach between the two interacting bodies was only 22 and $23 \mathrm{~nm}$ in the first and second sets of measurements, respectively $[60,61]$. Just this has made possible obtaining rather strong constraints on the Yukawa interaction in the range of the order of $10 \mathrm{~nm}$.

Measurements of the Casimir force in Ref. [57] have an advantage in that they were performed at even shorter separations starting from $10 \mathrm{~nm}$. The measurement data were compared with theoretical predictions of the Lifshitz theory and the extent of agreement between them has been quantified. This makes it possible to find the constraints on any hypothetical interaction which was not observed within the determined confidence interval. One more advantage of measurements at very short separations is that the comparison with theory in this case does not suffer from an uncertainty as to the correct description of free charge carriers in the Casimir effect. This unresolved problem (the so-called Casimir puzzle $[58,62]$ ) is of major importance at separations exceeding $100 \mathrm{~nm}$, but is immaterial at shorter separations where the Casimir force is completely determined by the bound (core) electrons.

We calculate the Yukawa-type force and the force due to two-axion exchange in the experimental configuration of Ref. [57] (note that an exchange of one pseudoscalar particle does not contribute in this case because the test bodies are not polarized). The constraints on the Yukawa interaction constant and the coupling constant of axion to nucleons are determined here at a 95\% confidence level. According to our results, in the interaction range from 1 to $3.7 \mathrm{~nm}$ the obtained constraints on the Yukawa interaction constant are up to a factor of $5 \times 10^{5}$ stronger than those obtained previously from measuring the lateral Casimir force between sinusoidally corrugated surfaces $[29,60,61]$. We show also that the constraints on a coupling constant of axionlike particles to nucleons, following from the experiment of Ref. [57], are stronger than those from measuring the lateral Casimir force $[51,60,61]$ for the axion masses satisfying a condition $m_{a}>17.8 \mathrm{eV}$. Within these interaction ranges, the stronger constraints on non-Newtonian gravity and coupling constant on axions to nucleons have been found only from the experiments on neutron scattering [37,38] and hydrogen beams [56], respectively.

The paper is organized as follows. In Sec. II we present a few necessary details of the experiment of Ref. [57] and the expressions for hypothetical forces in the experimental configuration. Section III contains the derivation of constraints on both non-Newtonian gravity and axionlike particles. In Sec. IV the reader will find our conclusions and the discussion of future prospects. We use the system of units in which $\hbar=c=1$.

\section{THE HYPOTHETICAL FORCES IN THE CASIMIR EXPERIMENT UTILIZING GOLD AND SILICON CARBIDE TEST BODIES}

In Ref. [57], the Casimir force $F_{C}$ between an $N$-doped SiC plate of $D=400 \mu \mathrm{m}$ thickness and a Au-coated borosilicate sphere of $R=10 \mu \mathrm{m}$ radius was measured in dry $\mathrm{N}_{2}$ atmosphere by means of an atomic force microscope within the separation region from $a=10 \mathrm{~nm}$ to $a=200 \mathrm{~nm}$. The thickness of a Au coating on the sphere was $d=100 \mathrm{~nm}$. The optical properties of the plate have been characterized by means of ellipsometers in the range of wavelengths from $140 \mathrm{~nm}$ to $30 \mu \mathrm{m}$ [63]. Together with the optical data for Au measured earlier [64], this allowed calculation of the Casimir force using the Lifshitz theory and comparison of theoretical results with the measurement data.

The most important for us are the force measurements at the shortest separations (the first five force magnitudes from 3.5 to $1.6 \mathrm{nN}$ were measured at separations $a$ from 10.8 to $14.4 \mathrm{~nm}$, respectively) and the measure of their agreement with theory. The dominant experimental error due to the error in determination of the cantilever spring constant $\Delta F_{C}^{\text {expt }}=0.35 \mathrm{nN}$ was independent of separation. Taking into account that the experimental separations were determined with an error $\Delta a \approx 1 \mathrm{~nm}$ [57], the theoretical forces calculated at the experimental separations were burdened by the error $\Delta F_{C}^{\text {theor }}=(2.48 \Delta a / a) F_{C}^{\text {theor }}$ which varies from 0.80 to $0.28 \mathrm{nN}$ within the separation interval 
indicated above. Note that both $\Delta F_{C}^{\text {theor }}$ and $\Delta F_{C}^{\text {expt }}$ have been determined at a $67 \%$ confidence level. Then, the halfwidth of the $95 \%$ confidence interval for the random quantity $F_{C}^{\text {theor }}-F_{C}^{\text {expt }}$ is defined as [62]

$$
\Theta_{0.95}(a)=2 \sqrt{\left[\Delta F_{C}^{\text {expt }}\right]^{2}+\left[\Delta F_{C}^{\text {theor }}\right]^{2}} .
$$

It is easily seen that $\Theta_{0.95}$ decreases from 1.8 to $0.9 \mathrm{nN}$ when the separation increases from 10.8 to $14.4 \mathrm{~nm}$. One can conclude that the magnitude of any hypothetical interaction, which was not observed in the experiment of Ref. [57], should satisfy the inequality

$$
\left|F^{\text {hyp }}(a)\right|<\Theta_{0.95}(a) \text {. }
$$

Now we list explicit expressions for the Yukawa-type force and for the force arising due to two-axion exchange between nucleons of a sphere and a plate. We start with the case of two atoms with masses $m_{1}$ and $m_{2}$ situated at the points $\boldsymbol{r}_{1}$ of a Au layer coating the sphere and $\boldsymbol{r}_{2}$ of a $\mathrm{SiC}$ plate. An exchange of a scalar particle of mass $M=1 / \lambda$ between these atoms, where $\lambda$ is the Compton wavelength of a particle, results in the Yukawa potential which is usually parametrized as a correction to Newton's gravitational potential [11]

$$
V_{\mathrm{Yu}}\left(r_{12}\right)=-\frac{G m_{1} m_{2}}{r_{12}} \alpha e^{-r_{12} / \lambda}
$$

Here, $G$ is the Newtonian gravitational constant, $\alpha$ is the dimensionless constant of Yukawa interaction, and $r_{12}=\left|\boldsymbol{r}_{1}-\boldsymbol{r}_{2}\right|$.

By integrating Eq. (3) over the volumes of a SiC plate (which can be safely considered as having an infinitely large area) and of a Au spherical envelope, and calculating the negative derivative with respect to the separation $a$, one arrives at the following expression for the force (see Refs. $[65,66]$ for details):

$$
\begin{aligned}
F_{\mathrm{Yu}}(a)= & -4 \pi^{2} G \alpha \rho_{1} \rho_{2} \lambda^{3} e^{-a / \lambda} \\
& \times\left[\Phi(R, \lambda)-\Phi(R-d, \lambda) e^{-d / \lambda}\right] .
\end{aligned}
$$

Here, $\rho_{1}=19.28 \mathrm{~g} / \mathrm{cm}^{3}$ and $\rho_{2}=3.21 \mathrm{~g} / \mathrm{cm}^{3}$ are the densities of $\mathrm{Au}$ and $\mathrm{SiC}$, respectively, and the function $\Phi$ is defined as

$$
\Phi(r, \lambda)=r-\lambda+(r+\lambda) e^{-2 r / \lambda} .
$$

Note that in Eq. (4) we disregarded the contribution of a borosilicate sphere core to the hypothetical force. Its account could lead to only a minor increase of $\left|F_{\mathrm{Yu}}\right|$ and, thus, to slightly stronger constraints on $\alpha$. We have also considered the $\mathrm{SiC}$ plate infinitely thick because in the region of $\lambda$ under consideration $\exp (-D / \lambda)=0$ at high precision.

We are coming now to two-axion exchange between the nucleons (protons and neutrons), which belong to a $\mathrm{Au}$ layer coating the sphere and a $\mathrm{SiC}$ plate, situated at the points $\boldsymbol{r}_{1}$ and $\boldsymbol{r}_{2}$, respectively. Assuming the pseudoscalar coupling between an axion and a nucleon, one obtains the following interaction potential $[12,13]$ :

$$
V_{a}\left(r_{12}\right)=-\frac{g_{a n}^{4}}{32 \pi^{3} m^{2}} \frac{m_{a}}{r_{12}^{2}} K_{1}\left(2 m_{a} r_{12}\right)
$$

where $g_{a n}$ is the dimensionless interaction constant of an axion to a nucleon (it is assumed that the interaction constants of an axion to a proton and a neutron are similar [13]), $m_{a}$ is the axion mass, $m$ is the mean nucleon mass, and $K_{1}(z)$ is the modified Bessel function of the second kind.

Integrating Eq. (6) over the volumes of a Au spherical layer and a $\mathrm{SiC}$ plate and performing the negative differentiation with respect to $a$, one obtains the following result (see Refs. [52,55] for details of the calculation):

$$
\begin{aligned}
F_{a}(a)= & -\frac{\pi}{2 m_{a} m^{2} m_{\mathrm{H}}^{2}} C_{1} C_{2} \int_{1}^{\infty} d u \frac{\sqrt{u^{2}-1}}{u^{3}} e^{-2 m_{a} u a} \\
& \times\left[\chi\left(R, m_{a} u\right)-\chi\left(R-d, m_{a} u\right) e^{-2 m_{a} u d}\right] .
\end{aligned}
$$

Here, $m_{\mathrm{H}}$ is the mass of atomic hydrogen,

$$
C_{i}=\rho_{i} \frac{g_{a n}^{2}}{4 \pi} \frac{Z_{i}+N_{i}}{\mu_{i}},
$$

where $i=1,2$ for Au and $\mathrm{SiC}$, respectively, $Z_{i}$ and $N_{i}$ are the number of protons and the mean number of neutrons, and $\mu_{i}=m_{i} / m_{\mathrm{H}}$, with $m_{i}$ being the mean atomic (molecular) mass. According to information presented in Ref. [11], one has

$$
\begin{array}{ll}
\frac{Z_{1}}{\mu_{1}}=0.40422, & \frac{N_{1}}{\mu_{1}}=0.60378, \\
\frac{Z_{2}}{\mu_{2}}=0.502701, & \frac{N_{2}}{\mu_{2}}=0.505708 .
\end{array}
$$

Finally, the function $\chi$ in Eq. (7) is defined as

$$
\chi(r, z)=r-\frac{1}{2 z}+\left(r+\frac{1}{2 z}\right) e^{-2 r z} .
$$

In the next section, the above expressions are used for constraining the parameters of Yukawa-type forces and axionlike particles from the measurement results of Ref. [57]. 


\section{CONSTRAINTS ON NON-NEWTONIAN GRAVITY AND AXIONLIKE PARTICLES}

To obtain constraints on the hypothetical forces of Yukawa-type, we have substituted the force $F_{\mathrm{Yu}}$ defined in Eqs. (4) and (5) in Eq. (2) in place of $F^{\text {hyp }}$. The obtained inequality was analyzed in the region of $a$ from 10.8 to $14.4 \mathrm{~nm}$. The constraints on $\alpha$ following from this inequality as a function of $\lambda$ are shown by line 2 in Fig. 1. In the same figure, the constraints on $\alpha$ found [29] from experiments on measuring the lateral Casimir force between sinusoidally corrugated surfaces $[60,61]$ are shown by line 1 .

As is seen in Fig. 1, the constraints of line 2 are stronger than those of line 1 within the range of $\lambda$ from 1 to $3.7 \mathrm{~nm}$. The largest strengthening by the factor of $495000 \approx$ $5 \times 10^{5}$ is reached at $\lambda=1 \mathrm{~nm}$. These are the strongest constraints for small $\lambda$ obtained to date from measuring the Casimir force. For even smaller $\lambda$, where the discrete structure of matter should be taken into account, the strongest constraints on the Yukawa-type interaction are obtained from atomic physics [67].

Within the interaction range $\lambda \geq 1 \mathrm{~nm}$ the constraints of line 2 are not the strongest ones. The strongest constraints on $\alpha$ obtained in this range so far follow from the experiments on neutron scattering. They are shown by lines 3 [37] and 4 [38]. The neutron scattering provides the strongest constraints on $\alpha$ within the interaction range up to $\lambda=10 \mathrm{~nm}$. For larger $\lambda$ the strongest constraints on $\alpha$ are shown by line 5 obtained [30] from measuring the normal Casimir force between sinusoidally corrugated surfaces $[68,69]$.

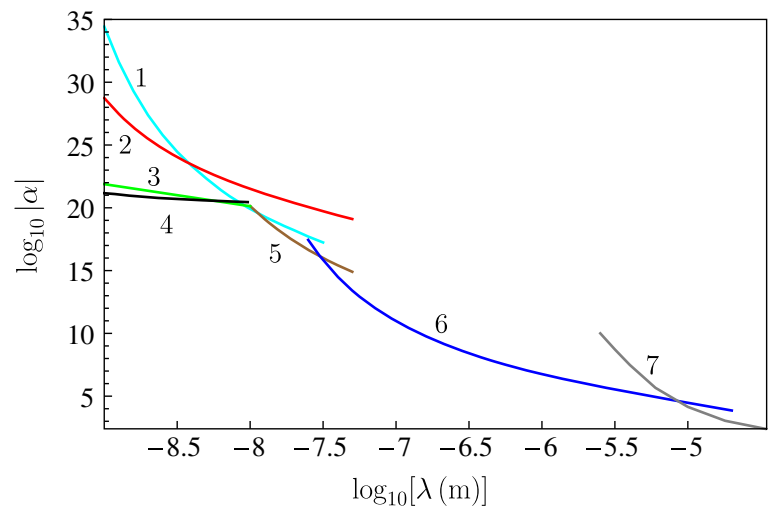

FIG. 1. Constraints on the strength of a Yukawa-type correction to Newton's gravitational law obtained from measuring the lateral Casimir force between corrugated surfaces (line 1), in this work from the experiment using a silicon carbide plate (line 2), from the experiments on neutron scattering (lines 3 and 4), from measuring the normal Casimir force between corrugated surfaces (line 5), from the Casimir-less experiment (line 6), and from the gravitational experiments (line 7) are shown as the functions of the interaction range (see the text for further discussion). The regions of the $(\lambda, \alpha)$-plane above each line are excluded and below each line are allowed by the experimental results.
Note that lines 1-4 have been obtained at a $95 \%$ confidence level, whereas line 5 was found at a $67 \%$ confidence level [30,68,69]. For even longer interactions $31 \mathrm{~nm}<\lambda<8 \mu \mathrm{m}$ the strongest constraints on $\alpha$ were obtained in the Casimir-less experiment of Ref. [31] (line 6), and in the interaction range $\lambda>8 \mu \mathrm{m}$ from the experiments of Cavendish type [19-22] (line 7). These two lines were obtained at a $95 \%$ confidence level. Recall that the regions of $(\lambda, \alpha)$-plane situated above each line in Fig. 1 are excluded by the results of the respective experiments, whereas the regions below each line are allowed.

As mentioned in Sec. I, rather strong constraints on $\alpha$ for small $\lambda$ were obtained [25] from measuring the Casimir force between two crossed cylinders [59]. These constraints, however, are not shown in Fig. 1 because they cannot be characterized by some definite confidence level. The point is that the Au films in the experiment of Ref. [59] were fixed to silica cylinders by means of a soft glue. Furthermore, the presence of a hydrocarbon organic layer on the interacting surfaces did not allow us to quantify the measure of agreement between experiment and theory [62].

We now turn our attention to the derivation of constraints on axionlike particles. For this purpose, in place of $F^{\text {hyp }}$ in Eq. (2) we substitute the force from Eqs. (7)-(10) arising due to two-axion exchange between the nucleons of a $\mathrm{Au}$ spherical layer and a SiC plate. The numerical analysis of the obtained inequality performed similar to the case of the Yukawa interaction leads to line 1 in Fig. 2 demonstrating the obtained constraints on $g_{a n}^{2} /(4 \pi)$ as a function of the axion mass $m_{a}$.

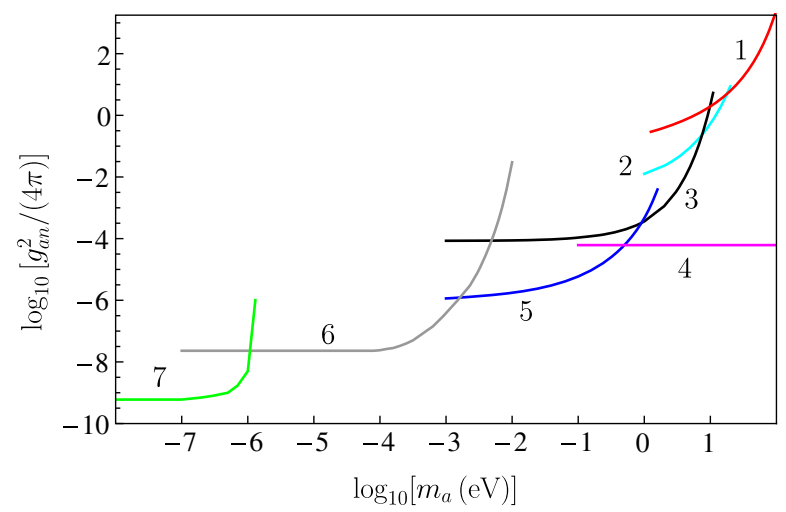

FIG. 2. Constraints on the coupling constant of axionlike particles to nucleons obtained in this work from the experiment using a silicon carbide plate (line 1), from measuring the lateral Casimir force between corrugated surfaces (line 2), from measuring the effective Casimir pressure (line 3), from the experiment using a beam of molecular hydrogen (line 4), from the Casimir-less experiment (line 5), from the gravitational experiments (line 6), and from the magnitometer measurements (line 7) are shown as the functions of the axion mass (see the text for further discussion). The regions of the $\left[m_{a}, g_{a n}^{2} /(4 \pi)\right]$-plane above each line are excluded and below each line are allowed by the experimental results. 
In the same figure, the constraints of line 2 were obtained in Ref. [51] from measuring the lateral Casimir force between sinusoidally corrugated surfaces $[60,61]$, and the constraints of line 3 were found in Ref. [50] from measuring the effective Casimir pressure [26,27]. Furthermore, lines 5 and 6 in Fig. 2 show the constraints derived [52] from the Casimir-less experiment of Ref. [31] and from the Cavendish-type experiments [46,47], respectively.

As is seen in Fig. 2, in the region of axion masses $m_{a}>$ $17.8 \mathrm{eV}$ the constraints of line 1 are stronger than all the above listed constraints obtained from the Casimir effect in the past. It should be noted, however, that line 4 obtained from the measure of agreement between experiment and theory for forces acting between protons in the beam of molecular hydrogen [56] (see also Ref. [70]) presents much stronger constraints for axion masses $m_{a}>0.5 \mathrm{eV}$. For heavier axions with $m_{a}>200 \mathrm{eV}$, further strengthening of constraints of Ref. [56] on $g_{\text {an }}$ was obtained in Ref. [70] by comparing the nuclear magnetic resonance experiment and theory for nucleons in deuterated molecular hydrogen. As to the lightest axions with masses $m_{a}<1 \mu \mathrm{eV}$, the strongest constraints on their coupling constant to nucleons were obtained from the magnetometer measurements using spin-polarized $\mathrm{K}$ and ${ }^{3} \mathrm{He}$ atoms [71] (see line 7). Similar to Fig. 1, the regions of the plane above each line are excluded and below each line are allowed by respective experiments. All the constraints under consideration have been obtained at a $95 \%$ confidence level.

\section{CONCLUSIONS AND DISCUSSION}

In this paper, we have obtained constraints on the Yukawatype corrections to Newtonian gravitational law and the coupling constant of axionlike particles to nucleons which follow from measuring the Casimir force between a $\mathrm{Au}$ coated microsphere and a silicon carbide plate. An advantage of this experiment operated in dry $\mathrm{N}_{2}$ atmosphere, as compared to more precise experiments operated in high vacuum, is that the force was measured down to $10 \mathrm{~nm}$ separation between the sphere and the plate. In spite of rather large experimental and theoretical errors, this allowed to obtain stronger constraints on the hypothetical interactions at sufficiently short distance range of the order of $1 \mathrm{~nm}$ (or for relatively large axion masses of more than $17.8 \mathrm{eV}$ ) than have been obtained previously from more precise measurements of the Casimir force performed at larger separation distances.

Specifically, in the interaction range $\lambda$ from 1 to $3.7 \mathrm{~nm}$ the constraints on the Yukawa interaction following from the experiment using a $\mathrm{SiC}$ plate are by up to a factor $5 \times 10^{5}$ stronger than those found previously from measuring the lateral Casimir force between sinusoidally corrugated surfaces. The constraints on the coupling constants of axionlike particles to nucleons following from the experiment using a $\mathrm{SiC}$ plate are stronger than those obtained from measuring the lateral Casimir force for the axion masses satisfying a condition $m_{a}>17.8 \mathrm{eV}$. It should be noted, however, that in these regions of relatively small $\lambda$ and large $m_{a}$ the strongest current constraints on non-Newtonian gravity and axion to nucleon coupling constant are provided not by the Casimir effect but by the neutron scattering and hydrogen beams, respectively.

It is pertinent to note that experiments on measuring the Casimir force have potentialities for obtaining stronger constraints on both non-Newtonian gravity and axionlike particles. Thus, as shown in Ref. [55], the constraints of Figs. 1 and 2 following from measuring the lateral and normal Casimir force between corrugated surfaces can be strengthened significantly by modifying the parameters of corrugations. Similar effect can be reached [55] by choosing thicker sectors of the patterned plate used in the Casimir-less experiment of Ref. [31]. One could mention also the recently proposed Casimir experiments at larger separation distances between the test bodies [72-75]. Several other very recent suggestions for searching the non-Newtonian gravity are based on measuring the Casimir-Polder interaction in the presence of a metallic shield [76], the normal mode splitting in the optomechanical cavity [77], and on precision spectroscopy of weakly bound molecules [78]. Taken together, these approaches should lead to further strengthening of the constraints on non-Newtonian gravity and axionlike particles.

\section{ACKNOWLEDGMENTS}

The work of G. L. K. and V. M. M. was partially supported by the Peter the Great Saint Petersburg Polytechnic University in the framework of the Program "5-100-2020." The work of P. K. was supported by the European Union through European Regional Development Fund CoE program Grant No. TK133 "The Dark Side of the Universe." V. M. M. was partially funded by Russian Foundation for Basic Research Grant No. 19-02-00453 A. His work was also partially supported by the Russian Government Program of Competitive Growth of Kazan Federal University. G. L.K. and V.M.M. are grateful to the Institute of Physics of the University of Tartu, where this work was performed, for the kind hospitality. 
[1] A. A. Anselm and N. G. Uraltsev, A second massless axion?, Phys. Lett. 114B, 39 (1982).

[2] S. Ferrara, J. Scherk, and B. Zumino, Algebraic properties of extended supergravity theories, Nucl. Phys. B121, 393 (1977).

[3] Y. Fujii, The theoretical background of the fifth force, Int. J. Mod. Phys. A 06, 3505 (1991).

[4] S. Deser and B. Zumino, Broken Supersymmetry and Supergravity, Phys. Rev. Lett. 38, 1433 (1977).

[5] S. Weinberg, A New Light Boson?, Phys. Rev. Lett. 40, 223 (1978).

[6] F. Wilczek, Problem of Strong $P$ and $T$ Invariance in the Presence of Instantons, Phys. Rev. Lett. 40, 279 (1978).

[7] J.E. Kim, Light pseudoscalars, particle physics and cosmology, Phys. Rep. 150, 1 (1987).

[8] S. Dimopoulos and G. F. Giudice, Macroscopic forces from supersymmetry, Phys. Lett. B 379, 105 (1996).

[9] J. Khoury and A. Weltman, Chameleon Fields: Awaiting Surprises for Tests of Gravity in Space, Phys. Rev. Lett. 93, 171104 (2004).

[10] J. Khoury and A. Weltman, Chameleon cosmology, Phys. Rev. D 69, 044026 (2004).

[11] E. Fischbach and C. L. Talmadge, The Search for NonNewtonian Gravity (Springer, New York, 1999).

[12] F. Ferrer and M. Nowakowski, Higgs- and Goldstone-bosonmediated long range forces, Phys. Rev. D 59, 075009 (1999).

[13] E. G. Adelberger, E. Fischbach, D. E. Krause, and R. D. Newman, Constraining the couplings of massive pseudoscalars using gravity and optical experiments, Phys. Rev. D 68, 062002 (2003).

[14] S. Aldaihan, D. E. Krause, J. C. Long, and W. M. Snow, Calculations of the dominant long-range, spin-independent contributions to the interaction energy between two nonrelativistic Dirac fermions from double-boson exchange of spin-0 and spin-1 bosons with spin-dependent couplings, Phys. Rev. D 95, 096005 (2017).

[15] E. G. Floratos and G. K. Leontaris, Low scale unification, Newton's law and extra dimensions, Phys. Lett. B 465, 95 (1999).

[16] A. Kehagias and K. Sfetsos, Deviations from $1 / r^{2}$ Newton law due to extra dimensions, Phys. Lett. B 472, 39 (2000).

[17] I. Antoniadis, N. Arkani-Hamed, S. Dimopoulos, and G. Dvali, New dimensions at a millimeter to a fermi and superstrings at a TeV, Phys. Lett. B 436, 257 (1998).

[18] N. Arkani-Hamed, S. Dimopoulos, and G. Dvali, Phenomenology, astrophysics, and cosmology of theories with millimeter dimensions and $\mathrm{TeV}$ scale quantum gravity, Phys. Rev. D 59, 086004 (1999).

[19] E. G. Adelberger, J. H. Gundlach, B. R. Heckel, S. Hoedl, and S. Schlamminger, Torsion balance experiments: A lowenergy frontier of particle physics, Prog. Part. Nucl. Phys. 62, 102 (2009).

[20] J. Chiaverini, S. J. Smullin, A. A. Geraci, D. M. Weld, and A. Kapitulnik, New Experimental Constraints on NonNewtonian Forces below $100 \mu \mathrm{m}$, Phys. Rev. Lett. 90, 151101 (2003).

[21] S. J. Smullin, A. A. Geraci, D. M. Weld, J. Chiaverini, S. Holmes, and A. Kapitulnik, Constraints on Yukawa-type deviations from Newtonian gravity at 20 microns, Phys. Rev. D 72, 122001 (2005).
[22] A. A. Geraci, S. J. Smullin, D. M. Weld, J. Chiaverini, and A. Kapitulnik, Improved constraints on non-Newtonian forces at 10 microns, Phys. Rev. D 78, 022002 (2008).

[23] V. A. Kuzmin, I. I. Tkachev, and M. E. Shaposhnikov, Restrictions imposed on light scalar particles by measurements of van der Waals forces, Pis'ma Zh. Eksp. Teor. Fiz. 36, 49 (1982) [JETP Lett. 36, 59 (1982)].

[24] V. M. Mostepanenko and I. Yu. Sokolov, The Casimir effect leads to new restrictions on long-range force constants, Phys. Lett. A 125, 405 (1987).

[25] V. M. Mostepanenko and M. Novello, Constraints on nonNewtonian gravity from the Casimir force measurements between two crossed cylinders, Phys. Rev. D 63, 115003 (2001).

[26] R. S. Decca, D. López, E. Fischbach, G. L. Klimchitskaya, D. E. Krause, and V.M. Mostepanenko, Tests of new physics from precise measurements of the Casimir pressure between two gold-coated plates, Phys. Rev. D 75, 077101 (2007).

[27] R. S. Decca, D. López, E. Fischbach, G. L. Klimchitskaya, D. E. Krause, and V. M. Mostepanenko, Novel constraints on light elementary particles and extra-dimensional physics from the Casimir effect, Eur. Phys. J. C 51, 963 (2007).

[28] M. Masuda and M. Sasaki, Limits on Nonstandard Forces in the Submicrometer Range, Phys. Rev. Lett. 102, 171101 (2009).

[29] V. B. Bezerra, G. L. Klimchitskaya, V. M. Mostepanenko, and C. Romero, Advances and prospects in constraining the Yukawa-type corrections to Newtonian gravity from the Casimir effect, Phys. Rev. D 81, 055003 (2010).

[30] G. L. Klimchitskaya, U. Mohideen, and V. M. Mostepanenko, Constraints on corrections to Newtonian gravity from two recent measurements of the Casimir interaction between metallic surfaces, Phys. Rev. D 87, 125031 (2013).

[31] Y.-J. Chen, W. K. Tham, D. E. Krause, D. López, E. Fischbach, and R. S. Decca, Stronger Limits on Hypothetical Yukawa Interactions in the 30-8000 nm Range, Phys. Rev. Lett. 116, 221102 (2016).

[32] W.-H. Tan, S.-Q. Yang, C.-G. Shao, J. Li, A.-B. Du, B.-F. Zhan, Q.-L. Wang, P.-S. Luo, L.-C. Tu, and J. Luo, New Test of the Gravitational Inverse-Square Law at Submillimeter Range with Dual Modulation and Compensation, Phys. Rev. Lett. 116, 131101 (2016).

[33] J. Wang, S. Guan, K. Chen, W. Wu, Z. Tian, P. Luo, A. Jin, S. Yang, C. Shao, and J. Luo, Test of non-Newtonian gravitational forces at micrometer range with two-dimensional force mapping, Phys. Rev. D 94, 122005 (2016).

[34] V. V. Nesvizhevsky and K. V. Protasov, Constraints on nonNewtonian gravity from the experiment on neutron quantum states in the Earth's gravitational field, Classical Quantum Gravity 21, 4557 (2004).

[35] H. Leeb and J. Schmiedmayer, Constraint on Hypothetical Light Interacting Bosons from Low-Energy Neutron Experiments, Phys. Rev. Lett. 68, 1472 (1992).

[36] A. Frank, P. van Isacker, and J. Gomez-Camacho, Probing additional dimensions in the Universe with neutron experiments, Phys. Lett. B 582, 15 (2004).

[37] V. V. Nesvizhevsky, G. Pignol, and K. V. Protasov, Neutron scattering and extra short range interactions, Phys. Rev. D 77, 034020 (2008). 
[38] Y. Kamiya, K. Itagami, M. Tani, G. N. Kim, and S. Komamiya, Constraints on New Gravitylike Forces in the Nanometer Range, Phys. Rev. Lett. 114, 161101 (2015).

[39] P. Brax and A.-C. Davis, Casimir, gravitational, and neutron tests of dark energy, Phys. Rev. D 91, 063503 (2015).

[40] Y. Xie, W.-T. Ni, P. Dong, and T.-Y. Huang, Second postNewtonian approximation of scalar-tensor theory of gravity, Adv. Space Res. 43, 171 (2009).

[41] M. Hohmann, L. Järv, P. Kuusk, and E. Randla, PostNewtonian parameters $\gamma$ and $\beta$ of scalar-tensor gravity with a general potential, Phys. Rev. D 88, 084054 (2013); Erratum, Phys. Rev. D89, 069901 (2014).

[42] B. Bertotti, L. Iess, and P. Tortora, A test of general relativity using radio links with the Cassini spacecraft, Nature (London) 425, 374 (2003).

[43] L. J. Rosenberg and K. A. van Bibber, Searches for invisible axions, Phys. Rep. 325, 1 (2000).

[44] M. Kawasaki and K. Nakayama, Axions: Theory and cosmological role, Annu. Rev. Nucl. Part. Sci. 63, 69 (2013).

[45] I. G. Ivastorza and J. Redondo, New experimental approaches in the search for axion-like particles, Prog. Part. Nucl. Phys. 102, 89 (2018).

[46] D. J. Kapner, T. S. Cook, E. G. Adelberger, J. H. Gundlach, B. R. Heckel, C. D. Hoyle, and H. E. Swanson, Tests of the Gravitational Inverse Square Law below the Dark-Energy Length Scale, Phys. Rev. Lett. 98, 021101 (2007).

[47] E. G. Adelberger, B. R. Heckel, S. Hoedl, C. D. Hoyle, D. J. Kapner, and A. Upadhye, Particle-Physics Implications of a Recent Test of the Gravitational Inverse-Square Law, Phys. Rev. Lett. 98, 131104 (2007).

[48] V. B. Bezerra, G. L. Klimchitskaya, V. M. Mostepanenko, and C. Romero, Constraints on the parameters of an axion from measurements of the thermal Casimir-Polder force, Phys. Rev. D 89, 035010 (2014).

[49] V. B. Bezerra, G. L. Klimchitskaya, V. M. Mostepanenko, and C. Romero, Stronger constraints on an axion from measuring the Casimir interaction by means of a dynamic atomic force microscope, Phys. Rev. D 89, 075002 (2014).

[50] V. B. Bezerra, G. L. Klimchitskaya, V. M. Mostepanenko, and $\mathrm{C}$. Romero, Constraining axion-nucleon coupling constants from measurements of effective Casimir pressure by means of micromachined oscillator, Eur. Phys. J. C 74, 2859 (2014).

[51] V. B. Bezerra, G. L. Klimchitskaya, V. M. Mostepanenko, and C. Romero, Constraints on axion-nucleon coupling constants from measuring the Casimir force between corrugated surfaces, Phys. Rev. D 90, 055013 (2014).

[52] G. L. Klimchitskaya and V. M. Mostepanenko, Improved constraints on the coupling constants of axion-like particles to nucleons from recent Casimir-less experiment, Eur. Phys. J. C 75, 164 (2015).

[53] V. B. Bezerra, G. L. Klimchitskaya, V. M. Mostepanenko, and C. Romero, Constraining axion coupling constants from measuring the Casimir interaction between polarized test bodies, Phys. Rev. D 94, 035011 (2016).

[54] G. L. Klimchitskaya and V. M. Mostepanenko, Constraints on axionlike particles and non-Newtonian gravity from measuring the difference in Casimir forces, Phys. Rev. D 95, 123013 (2017).
[55] G. L. Klimchitskaya, Recent breakthrough and outlook in constraining the non-Newtonian gravity and axion-like particles from Casimir physics, Eur. Phys. J. C 77, 315 (2017).

[56] N. F. Ramsey, The tensor force between two protons at long range, Physica (Amsterdam) 96A, 285 (1979).

[57] M. Sedighi, V. B. Svetovoy, and G. Palasantzas, Casimir force measurements from silicon carbide surfaces, Phys. Rev. B 93, 085434 (2016).

[58] G. L. Klimchitskaya, U. Mohideen, and V. M. Mostepanenko, The Casimir force between real materials: Experiment and theory, Rev. Mod. Phys. 81, 1827 (2009).

[59] T. Ederth, Template-stripped gold surfaces with 0.4-nm rms roughness suitable for force measurements: Application to the Casimir force in the 20-100-nm range, Phys. Rev. A 62, 062104 (2000).

[60] H.-C. Chiu, G. L. Klimchitskaya, V. N. Marachevsky, V. M. Mostepanenko, and U. Mohideen, Demonstration of the asymmetric lateral Casimir force between corrugated surfaces in nonadditive regime, Phys. Rev. B 80, 121402(R) (2009).

[61] H.-C. Chiu, G. L. Klimchitskaya, V. N. Marachevsky, V. M. Mostepanenko, and U. Mohideen, Lateral Casimir force between sinusoidally corrugated surfaces: Asymmetric profiles, deviations from the proximity force approximation, and comparison with exact theory, Phys. Rev. B 81, 115417 (2010).

[62] M. Bordag, G. L. Klimchitskaya, U. Mohideen, and V. M. Mostepanenko, Advances in the Casimir Effect (Oxford University Press, Oxford, 2015).

[63] M. Sedighi, V. B. Svetovoy, W. H. Broer, and G. Palasantzas, Casimir forces from conductive silicon carbide surfaces, Phys. Rev. B 89, 195440 (2014).

[64] V. B. Svetovoy, P. J. van Zwol, G. Palasantzas, and Th. M. De Hosson, Optical properties of gold films and the Casimir force, Phys. Rev. B 77, 035439 (2008).

[65] R. S. Decca, E. Fischbach, G. L. Klimchitskaya, D. E. Krause, D. López, and V. M. Mostepanenko, Application of the proximity force approximation to gravitational and Yukawa-type forces, Phys. Rev. D 79, 124021 (2009).

[66] E. Fischbach, G. L. Klimchitskaya, D. E. Krause, and V. M. Mostepanenko, On the validity of constraints on light elementary particles and extra-dimensional physics from the Casimir effect, Eur. Phys. J. C 68, 223 (2010).

[67] M. S. Safronova, D. Budker, D. DeMille, D. F. Jackson Kimball, A. Derevianko, and C. W. Clark, Search for new physics with atoms and molecules, Rev. Mod. Phys. 90, 025008 (2018).

[68] A. A. Banishev, J. Wagner, T. Emig, R. Zandi, and U. Mohideen, Demonstration of Angle-Dependent Casimir Force between Corrugations, Phys. Rev. Lett. 110, 250403 (2013).

[69] A. A. Banishev, J. Wagner, T. Emig, R. Zandi, and U. Mohideen, Experimental and theoretical investigation of the angular dependence of the Casimir force between sinusoidally corrugated surfaces, Phys. Rev. B 89, 235436 (2014).

[70] M. P. Ledbetter, M. V. Romalis, and D. F. Jackson Kimball, Constraints on Short-Range Spin-Dependent Interactions 
from Scalar Spin-Spin Coupling in Deuterated Molecular Hydrogen, Phys. Rev. Lett. 110, 040402 (2013).

[71] G. Vasilakis, J. M. Brown, T. R. Kornak, and M. V. Romalis, Limits on New Long Range Nuclear Spin-Dependent Forces Set with a K- ${ }^{3} \mathrm{He}$ Comagnetometer, Phys. Rev. Lett. 103, 261801 (2009).

[72] A. Almasi, Ph. Brax, D. Iannuzzi, and R. I. P. Sedmik, Force sensor for chameleon and Casimir force experiments with parallel-plate configuration, Phys. Rev. D 91, 102002 (2015).

[73] G. L. Klimchitskaya, V. M. Mostepanenko, R. I. P. Sedmik, and H. Abele, Prospects for searching thermal effects, nonNewtonian gravity and axion-like particles: CANNEX test of the quantum vacuum, Symmetry 11, 407 (2019).

[74] G. L. Klimchitskaya, V. M. Mostepanenko, and R. I. P. Sedmik, Casimir pressure between metallic plates out of thermal equilibrium: Proposed test for the relaxation properties of free electrons, Phys. Rev. A 100, 022511 (2019).

[75] G. Bimonte, G. L. Klimchitskaya, and V. M. Mostepanenko, Universal experimental test for the role of free charge carriers in the thermal Casimir effect within a micrometer separation range, Phys. Rev. A 95, 052508 (2017).

[76] R. Bennett and D. H. J. O'Dell, Revealing short-range non-Newtonian gravity through Casimir-Polder shielding, New J. Phys. 21, 033032 (2019).

[77] J. Liu and K.-D. Zhu, Detecting large extra dimensions with optomechanical levitated sensors, Eur. Phys. J. C 79, 18 (2019).

[78] M. Borkowski, A. A. Buchachenko, R. Ciuryło, P. S. Julienne, H. Yamada, Y. Kikuchi, Y. Takasu, and Y. Takahashi, Weakly bound molecules as sensors of new gravitylike forces, Sci. Rep. 9, 14807 (2019). 